\title{
A unique milestone
}

Cite as: CMAJ 2021 March 8;193:E345-6. doi: 10.1503/cmaj.202569

W e all mark milestones in our lives: we graduate, we marry, have children, retire.

This week marked a unique milestone in my medical career, a milestone that was unthinkable when I graduated in 1976. Back then, I was a young and idealistic physician, intent on curing illness and saving lives. I never would have predicted where I now find myself, reflecting on the hundredth time that I have provided medical assistance in dying (MAiD) to a suffering patient.

When I began medical practice in 1977 , in a small farming community in Prince Edward Island, I discovered the joy of family medicine, including delivering babies, looking after young families and caring for older adults. I soon realized, though, that my ideals of curing illness and saving lives were not always achievable. I witnessed deaths in patients of all ages and from all causes, including suicides, traumatic farm injuries, motor vehicle accidents, cancers and heart disease. I observed - and likely provided - unnecessary treatment that only prolonged the dying. I have unpleasant memories of patients near death with intravenous antibiotics flowing, yet with uncontrolled pain and dyspnea. Opiates were still a feared entity and there was not adequate home care to support death at home. It soon became evident to me that an important part of family practice involved not only helping patients to live and to live well, but also to die and to die well.

Around this time, the palliative care movement started in Canada. In the 1970s, it was in its infancy but thanks to pioneers like Dr. Balfour Mount, there evolved a philosophy in which death was not necessarily the enemy. Acceptance of impending mortality and the avoidance of unnecessary interventions could improve the quality of life - and of death - for those with incurable illness.

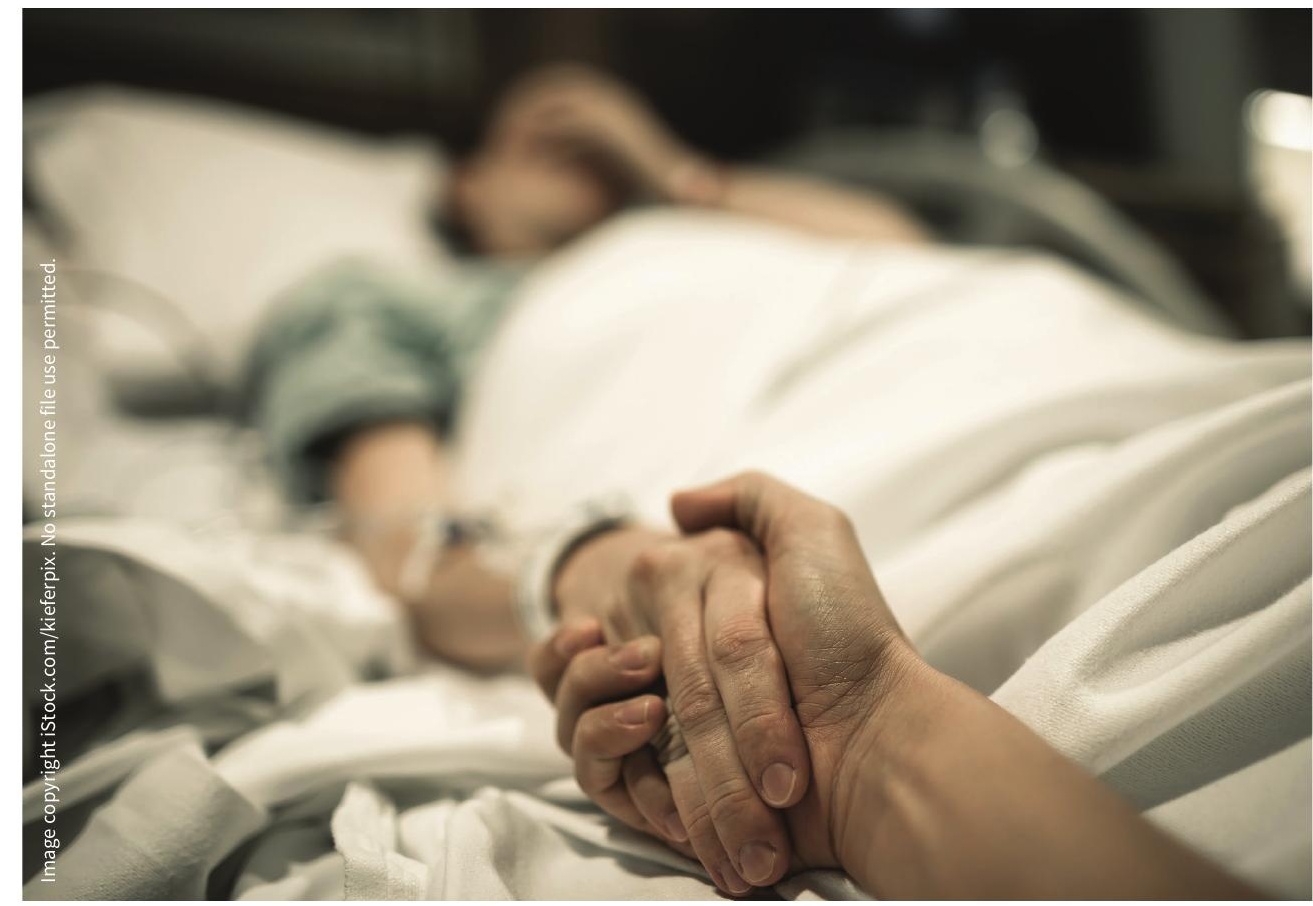

Protocols were developed to control pain, nausea and shortness of breath. Efforts were made to prepare patients and families for death and to assuage their existential and spiritual fears.

This philosophy captivated me. My experiences with dying patients convinced me that Dr. Mount was correct. Hearing him speak so eloquently and with such passion at palliative care conferences further inspired me. I became a "disciple" and was eager to involve other physicians. This was not always easy, as many had difficulty withdrawing treatment and discussing death with patients and their families. Often it was less troublesome to continue "curative" treatment, even though it might have been futile. At this point, the medical world had not yet embraced the concept of a "good death."

I moved to Brockville, Ontario, in 1983 and was excited to discover that our local hospital had recently established a palliative care program with designated palliative care beds, as well as a community outreach team. I joined the team, which continues to provide exemplary palliative care.

I have cared for hundreds of terminally ill patients, and for the most part they had "good deaths." The "bad deaths," be they "bad" because of uncontrolled pain or confusion and delirium, were difficult for patients and families to bear and for caregivers to witness. When we set out on a path with a patient in palliative care and their family, we commit to providing a safe and peaceful journey. We are disappointed when we fail.

In the 1990s, palliative sedation became more common. It allowed for the induction of deep sedation in patients who were suffering intolerably in the last two weeks of their lives. It could take days before they died, but at least it was an improvement over uncontrolled delirium. They died a "natural" death. 
Eventually I began to realize that despite our best care and efforts, a small number of patients continued to suffer. Although good palliative care could control their physical suffering, these patients were not willing to tolerate the loss of independence, the overwhelming fatigue, the loss of meaning in their lives, and the loss of enjoyment of food and its resulting cachexia. They did not want to be a burden on their families or society. A few asked if there was something that we could do to expedite their deaths. We could not consider these requests and redoubled our efforts to provide effective palliative care.

Unfortunately, some took matters into their own hands. I have had patients with cancer who took overdoses of medication, and another who died from a selfinflicted gunshot wound. We had failed these patients and their families. It caused me to wonder: Was there a better way for these individuals? As a result, I became an advocate for MAiD. Although my passion for palliative care had not diminished, I could not abandon the small number of patients who could not abide continuing to suffer.

After the passage of Bill C-14 in 2016, MAiD became legal. Within a month, a man with amyotrophic lateral sclerosis requested my help. He could not tolerate his loss of independence and of speech and his declining physical abilities. He was fed through a gastric tube and had declined respiratory support. He clearly met all eligibility criteria. I was apprehensive on that last visit to his home, but his grace and gratitude put me at ease. The tranquility of his death, and of all the MAiD deaths I've attended, was so reassuring. Four years later, my hundredth patient was a man with metastatic cancer to his cervical spine and cord compression. The other 98 souls suffered from cancer, heart disease, pulmonary disease or degenerative neurologic disease.

In these four years, I have assessed close to 150 patients for eligibility for an assisted death. Some were not eligible. Those who were had access to good palliative care and had loving families and supportive partners. Although the majority chose not to suffer until the end, there were many who ultimately did not choose MAiD, for a variety of reasons. Some lost competence or died during the reflection period. Others never experienced intolerable suffering, often thanks to excellent palliative care. All were appreciative that the option was available to them.

I will always remember these 100 brave and determined people and their families. I am thankful that they let me share with them this intimate and sacred moment. They have taught me about courage, conviction and independence. Their families have taught me about love and loyalty.

We have made great progress in endof-life care in these past 50 years. There is still much to be done. Good palliative care should be available to all. MAiD is a right of those few who can no longer tolerate suffering. Why can't MAiD be integrated into all palliative care programs (it would comprise a small part)? My hope is that physicians, both generalists and specialists, will see MAiD as the act of mercy and respect that it truly is. Although only a small number of our patients will request it ( $1 \%-2 \%$ of all people who die), their voices must be heard and their suffering legitimized.

\section{Gerald Patrick Ashe MD}

Brockville, Ont.

This article has been peer reviewed.

This is a true story. Next of kin of depicted patients have given consent.

Content licence: This is an Open Access article distributed in accordance with the terms of the Creative Commons Attribution (CC BY-NC-ND 4.0) licence, which permits use, distribution and reproduction in any medium, provided that the original publication is properly cited, the use is noncommercial (i.e., research or educational use), and no modifications or adaptations are made. See: https://creativecommons.org/ licenses/by-nc-nd/4.0/ 